\title{
Mid-Circumflex Artery
}

National Cancer Institute

\section{Source}

National Cancer Institute. Mid-Circumflex Artery. NCI Thesaurus. Code C102326.

The segment of the left circumflex artery between the first and second marginal branches. 